\title{
Unexplained Fatigue in an Otherwise Healthy Man Linked to Kikuchi- Fujimoto Disease, A Case Report
}

\author{
Vivek Pant ${ }^{1}$, Santosh Pradhan ${ }^{1}$, Vijay Kumar Sharma ${ }^{2}$ \\ ${ }^{1}$ Department of Clinical Biochemistry, Samyak Diagnostic Lalitpur, Nepal \\ ${ }^{2}$ Department of Clinical Biochemistry, Institute of Medicine, Tribhuvan University Teaching hospital, Kathmandu, Nepal
}

\section{ABSTRACT}

\begin{abstract}
Kikuchi histiocytic necrotizing lymphadenitis is a benign and self-limited illness usually characterized by cervical lymphadenopathy and fever. We present a case of a 42-year male who complained of extreme fatigue for 2 weeks. On laboratory workup, he had leucopenia and thrombocytopenia with normal peripheral blood and bone marrow examination. The radiological investigation revealed multiple enlarged lymph nodes in the left axilla and left supraclavicular region. The subsequent excisional biopsy of the axillary node clinched the diagnosis of Kikuchi- Fujimoto disease. The patient was completely recovered and laboratory parameters were normal with supportive treatment. Kikuchi- Fujimoto disease should be considered in patients with unexplained fatigue with lymphadenopathy and early biopsy prevents unnecessary investigations as well as potentially harmful treatments.
\end{abstract}

Keywords: Kikuchi- Fujimoto disease; Lymphadenopathy; Unexplained fatigue

\section{Correspondence:}

Dr. Vivek Pant, MD Biochemistry, Department of Clinical Biochemistry, Samyak Diagnostic; Jawalakhel, Lalitpur, Nepal ORCID ID: 0000-0002-3967-1851

Email: drvpant@gmail.com

Submitted: $10^{\text {th }}$ June 2021 Accepted: $25^{\text {th }}$ June 2021

Source of Support: None

Conflict of Interest: No

Citation: Pant V, Pradhan S, Sharma VK. Unexplained Fatigue in an Otherwise Healthy Man Linked to KikuchiFujimoto Disease, A Case Report. NMJ 2021;4(1):454-6. DOI 10.3126/nmj.v4i1.38222

\section{INTRODUCTION}

Kikuchi- Fujimoto disease (KFD) is a rare benign inflammatory disorder of unknown etiology. Typical clinical features include fever, anterior cervical lymphadenopathy, and night sweats. The hallmark histopathological finding is necrotizing lymphadenitis with the absence of neutrophils. ${ }^{1}$ It is common in Asia and the affected individuals are mostly younger females. ${ }^{1-3}$ The exact cause of KFD is unknown but viral or autoimmune etiology has been reported. ${ }^{4}$ The role of Epstein-Barr virus (EBV), as well as other viruses, in the pathogenesis of KFD remains controversial. The incidence of Human leukocyte antigen (HLA) class II genes, DPA $1 * 01$, and DPB $1 * 0202$ alleles is higher in patients with KFD than in healthy control subjects. ${ }^{5}$ These genes are extremely rare among Caucasians but common in Asian people. ${ }^{5}$ In a study that described 244 patients with KFD, the most common signs and symptoms seen were lymphadenopathy in $100 \%$, fever in $35 \%$, rash in $10 \%$, arthritis in $7 \%$, fatigue in $7 \%$, and hepatosplenomegaly in $3 \%$ of $^{\text {cases. }}{ }^{6}$

Thus, KFD may rarely present with nonspecific symptoms like weakness and lymphadenitis in sites other than the cervical region. This is often confused with several other conditions like tuberculosis, EBV infection, Human Immunodeficiency (HIV) virus, Systemic lupus erythematosus (SLE), and malignancy. Here, we present a case of an adult male who complained of weakness that was unexplained and received numerous laboratory investigations before the final diagnosis of KFD was made. 


\section{\# CASE REPORT}

A 42 years male visited the medical outpatient department with complaints of fatigue and extreme weakness of the lower leg bilaterally for two weeks. The patient also complained of lowgrade fever. No significant finding on general and systemic examination was noted. His laboratory investigation revealed leucopenia and thrombocytopenia. Total count was $2,300 / \mathrm{mm}^{3}$ [N:48 L:38 M:8 E:6 B:0], hemoglobin was $15.5 \mathrm{gm} / \mathrm{dl}$ and platelet count was $72,000 / \mathrm{mm}^{3}$. Organ function tests (liver, kidney, and thyroid) were all within normal limits. C-reactive protein (CRP) was increased to $13.6 \mathrm{mg} / \mathrm{L}(<6.0)$ and erythrocyte sedimentation rate (ESR) was increased to $23 \mathrm{~mm} /$ hour $(0-12 \mathrm{~mm} /$ hour). Dengue IgG/IgM antibody was negative. Malaria parasite antigen was negative. Serum lactate dehydrogenase, 8 am cortisol level and creatinine phosphokinase were normal. Peripheral blood smear revealed cytopenia. Vitamin $\mathrm{B}_{12}$, folic acid, and serum iron were also normal. Bone marrow aspiration demonstrated markedly hypercellular marrow showing no abnormal cells, parasites, or granulomas. HIV and viral hepatitis screening were negative. The patient was not taking any herbal preparations and had not any recent travel history. He did not have any specific complaints besides weakness. Tuberculosis was ruled out. Work up for systemic lupus erythematosus and other autoimmune diseases were negative. Investigation for EBV capsid antigen and nuclear antigen and cytomegalovirus were negative. All these investigations were done because the patient had a progressive increasing weakness, and leukopenia with thrombocytopenia was deteriorating. Throughout these periods of investigations, the patient was being managed with prophylactic antibiotics.

The case was then discussed with other medical and surgical faculties. The contrast CT -scan of the chest and abdomen was done that revealed multiple enlarged lymph nodes in the left axilla and left the supraclavicular region with the largest $18 \times 12$ $\mathrm{mm}$ in size. Then, an excisional biopsy of six lymph nodes from the left axillary and supraclavicular area was done which showed several aggregates of histiocytes in the interfollicular area. A single necrotic focus with apoptotic debris devoid of neutrophils was seen in the largest node. No fungal elements were seen. Molecular genetic assay in tissue biopsy specimen for identification of resistance to rifampicin was not detected. No organisms were isolated after 48 hours of incubation of the lymph node. The immunohistochemistry study did not reveal any abnormality. The diagnosis of KFD was made based on the finding of a lymph node biopsy. The patient was then managed with paracetamol and multivitamins. He was advised to have complete bed rest and high-energy diets. On follow-up, after two weeks patient had no complaints and his blood counts were in the normal range. Informed consent was taken from the patient for publication of this case report.

\section{DISCUSSION}

KFD may present with nonspecific symptoms like weakness and viral flu-like prodrome, so several diseases such as tuberculosis, EBV infection, HIV, SLE, and malignancy should be considered in the differential diagnosis. Work out for EBV, CMV, HIV, SLE, tuberculosis and hematological malignancy was negative in our case. Around $25-58 \%$ of patients experience leukopenia and $2-5 \%$ of patients experience leukocytosis in KFD. ${ }^{7}$ In the index case leukopenia and thrombocytopenia were seen and the severity increased day by day. The total leucocyte count was $2100 / \mathrm{mm}^{3}$ and the total platelet count was $45,000 / \mathrm{mm}^{3}$ when it reached its lowest value.

Histopathological findings in KFD include partially preserved nodal architecture with the expansion of the paracortex by patchy areas of fibrinoid necrosis with marked apoptosis and nuclear debris, surrounded by aggregates of histiocytes with crescentic nuclei, activated T-lymphocytes (immunoblasts), and characteristic absence of neutrophils and eosinophils. ${ }^{4,7}$ In the index case, excisional biopsy of the left axillary node demonstrated reactive and lymphoid follicles with a single necrotic focus with apoptosis, devoid of neutrophils. In KFD, CD30 positive cytotoxic $\mathrm{T}$ cells are abundant around necrotic areas which help to differentiate it from SLE. ${ }^{8}$ In immunohistochemistry, few immunoblasts around the necrotic area were positive for CD30 in this case.

Since the disease is a self-limited, only symptomatic treatment to relieve distressing complaints should be used like analgesics, antipyretics, and rest. The patient, in this case, was managed with supportive measures. KFD spontaneously resolves within 1-4 months; however, $3-4 \%$ of patients will experience recurrent episodes of KFD. ${ }^{9}$ Reoccurrence has been recorded after a period of 2-10 years of initial presentation. ${ }^{10}$

Various atypical presentation or association of KFD has been reported such as high levels of circulating Epstein-Barr viral DNA $^{11}$, lymphocytic meningitis, ${ }^{12}$ autoimmune hemolytic anemia, ${ }^{13}$ Corona virus disease-19, ${ }^{14}$ relapsing polychondritis, ${ }^{15}$ acute appendicitis, ${ }^{16}$ periportal and retroperitoneal lymphadenopathy, ${ }^{17}$ fever of unknown origin,,${ }^{18}$ and acute renal failure. ${ }^{19}$ The consequence of various nonspecific clinical presentations of KFD is a broader differential diagnosis. Thus, it is easily mistaken for lymphoma, tuberculosis, viral diseases, and autoimmune diseases. The index case also suffered a wide range of investigations and tolerated unnecessary antibiotics treatment and hospital stay. Proper physical examination for identification of any enlarged lymph nodes and early biopsy in suspected cases prevents unnecessary investigations as well as iatrogenic effects.

\section{CONCLUSIONS}

KFD although is rare, should be considered in the differential diagnosis of chronic fatigue and lymphadenopathy in otherwise normal cases. An early biopsy in suspected cases prevents unnecessary investigations as well as potentially harmful treatments. Scientific reporting of Kikuchi-Fujimoto disease may lead to more recognition by healthcare providers and the prevention of unnecessary investigations. 


\section{REFERENCES}

1. Dorfman RF. Histiocytic necrotizing lymphadenitis of Kikuchi and Fujimoto. Archives of pathology \& laboratory medicine. 1987;111(11):1026-9. Website

2. Yen A, Fearneyhough P, Raimer SS, Hudnall SD. EBV-associated Kikuchi's histiocytic necrotizing lymphadenitis with cutaneous manifestations. Journal of the American Academy of Dermatology. 1997;36(2):342-6. Website

3. Dorfman RF, Berry GJ. Kikuchi's histiocytic necrotizing lymphadenitis: an analysis of 108 cases with emphasis on differential diagnosis. In Seminars in diagnostic pathology 1988; 5(4):329-45. $\underline{\text { Website }}$

4. Melıkoglu MA, Melıkoglu M. The clinical importance of lymphadenopathy in systemic lupus erythematosus. Acta reumatologica portuguesa. 2008;33(4):402-6. Website

5. Tanaka T, Ohmori M, Yasunaga S, Ohshima K, Kikuchi M, Sasazuki T. DNA typing of HLA class II genes (HLA-DR, -DQ andDP) in Japanese patients with histiocytic necrotizing lymphadenitis (Kikuchi's disease). HLA. 1999;54(3):246-53 Website

6. Kucukardali Y, Solmazgul E, Kunter E, Oncul O, Yildirim S, Kaplan M. Kikuchi-Fujimoto disease: analysis of 244 cases. Clinical rheumatology. 2007;26(1):50-4. Website

7. Qadri F, Atkin GK, Thomas D, Das SK. Kikuchi's disease: an important cause of cervical lymphadenopathy. Clinical medicine. 2007;7(1):82-4. Website

8. Tabata T, Takata K, Miyata-Takata T, Sato Y, Ishizawa S, Kunitomo T, et al. Characteristic Distribution Pattern of CD30-positive Cytotoxic T Cells Aids Diagnosis of Kikuchi-Fujimoto Disease. Applied Immunohistochemistry and Molecular Morphology. 2016;26(4):274-282. Website

9. Yilmaz M, Camci C, Sari I, Okan V, Sevinc A, Onat AM, Buyukhatipoglu H. Histiocytic necrotizing lymphadenitis (KikuchiFujimoto's disease) mimicking systemic lupus erythematosus: a review of two cases. Lupus. 2006;15(6):384-7.

10. Aano S, Akaike Y, Muramatsu T, Wakasa H, Yoshida H, Kondou $R$, et al. Necrotizing lymphadenitis: a clinicopathological and immunohistochemical study of four familial cases and five recurrent cases. Virchows Archiv A. 1991;418(3):215-23. Website
11. Moyer A, Hanafi MZ, Scordino T, Bronze M. Kikuchi-Fujimoto disease: an atypical presentation of a rare disease. Cureus. 2019;11(2): e3999. Website

12. Meni C, Chabrol A, Wassef M, Gautheret-Dejean A, Bergmann JF, Mouly S. An atypical presentation of Kikuchi-Fujimoto disease. La Revue de medecine interne. 2012;34(6):373-6. Website

13. Halawa AR, Ahmad MK, Nashwan AJ. An atypical presentation of Kikuchi-Fujimoto disease: A case report \& literature review. Clinical Case Reports. 2020;8(12):3515-9. Website

14. Stimson L, Stitson R, Bahhadi-Hardo M, Renaudon-Smith E. COVID-19 associated Kikuchi-Fujimoto disease. $\mathrm{Br} J$ Haematol. 2021;192(5):e124-e126. Website

15. Mahajan T, Merriman RC, Stone MJ. Kikuchi-Fujimoto disease (histiocytic necrotizing lymphadenitis): report of a case with other autoimmune manifestations. In Baylor University Medical Center Proceedings. Taylor \& Francis. 2007;20 (2):149-51. Website

16. Pandey V, Khatib Y, Pandey R, Khade AL, Khare M. KikuchiFujimoto disease masquerading as acute appendicitis. Journal of clinical and diagnostic research: JCDR. 2017;11(6):ED26. Website

17. Vu D, Reddy S, Day L, Aydin N, Misra S. Kikuchi-Fujimoto disease: unusual presentation of rare disease. Clinics and practice. 2016;6(1):16-7. Website

18. Ranjan P, Soneja M, Subramonian NK, Kumar V, Ganguly S, Kumar T, Singh G. Fever of unknown origin: an unusual presentation of kikuchi-fujimoto disease. Case reports in immunology. 2015; 2015. Website

19. Da Silva AF, Focaccia R, De Oliveira AC, Sementilli A, Reis GF. Kikuchi-Fujimoto disease: an unusual association with acute renal failure. The Brazilian Journal of Infectious Diseases. 2010 ;14(6):621-7. Website 